\title{
Influence of a protocol of Pilates exercises on the contractility of the pelvic floor muscles of non-institutionalized elderly persons
}

Ligia Muniz de Souza'

Ana Beatriz Gomes de Souza Pegorare Gustavo Christofoletti',2 Suzi Rosa Miziara Barbosa1,3

\section{Abstract}

Objective: To investigate the influence of a protocol of Pilates exercises on the functionality and contractility of the pelvic floor muscles (PFM) of older women living in the city of Campo Grande, Mato Grosso, Brazil. Method: Ten women (median age of $63.4 \pm 4.5$ years) with little or no pelvic floor dysfunction were subjected to 24 sessions of Pilates exercises lasting one hour each, for 12 weeks. The pressure of the pelvic floor muscles (PFM) was assessed using a perineometer $\left(\mathrm{cmH}_{2} \mathrm{O}\right)$ and contractility was assessed with the PERFECT scheme. Data were described as median \pm interquartile range and inferential analysis was performed using the Wilcoxon paired test, with a significance level of 5\%. Results: In view of the proposed treatment, the degree of voluntary contraction of the PFM of the participants increased from $79.0 \pm 83.5$ to $90.0 \pm 82.0 \mathrm{cmH}_{2} 0$, with a statistically significant difference in paired comparison $(p=0.012)$. According to the PERFECT scheme, there was an increase in contraction time (from $5.0 \pm 0.1$ to $7.0 \pm 4.7$ seconds) and in the number of fast repetitions (from $7.0 \pm 4.5$ to $8.0 \pm 4.7$ ), with a statistically significant difference for both variables ( $p=0.017$ and $p=0.008$, respectively). Conclusion: The results indicate that the Pilates method increased the contractility and pressure of the PFM of elderly women with little or no PFM impairment. Further studies are required to determine whether the Pilates method is an effective method for the treatment of women with severe pelvic floor dysfunction.

\footnotetext{
Universidade Federal de Mato Grosso do Sul, Instituto Integrado de Saúde INISA, Curso de Fisioterapia. Campo Grande, Mato Grosso do Sul, Brasil.

2 Universidade Federal de Mato Grosso do Sul, Programa de Pós Graduação em Saúde e Desenvolvimento da região Centro-Oeste. Campo Grande, Mato Grosso do Sul, Brasil.

3 Universidade Federal de Mato Grosso do Sul, Universidade Aberta à Pessoa Idosa. Campo Grande, Mato Grosso do Sul, Brasil.
}

Keywords: Aged. Exercise Movement Techniques. Physical Therapy Modalities. 


\section{INTRODUCTION}

Pilates emerged as a method of rehabilitation during World War I, when Joseph Hubertus Pilates applied his knowledge to rehabilitate injured men. The popularity of the method grew most in the $1980 \mathrm{~s}^{1,2}$. More recently, Pilates has been used by health care professionals to integrate the mind and body of subjects, resulting in improved fitness (flexibility, strength and balance) and body consciousness. The method features ground and apparatus-based exercises created by Joseph Pilates ${ }^{1-4}$.

There are six key principles involved in Pilates: concentration, control, precision, fluidity of movement, breathing and center of strength ${ }^{1,3,5}$. The center of strength, also called the "core", "power house" or "engagement" refers to the region of specific groups of muscles (anterior abdominal wall, spinal extensors, hip extensors, hip flexors and pelvic floor muscles).

The pelvic floor (PF) consists of muscles, fascia and ligaments that occupy the region of the lower pelvis. These muscles are classified as skeletal striated or voluntary contraction muscles, and thus respond to training techniques just like the other skeletal muscles of the human body. There are two hypotheses about the mechanisms by which the strengthening of this muscle group can prevent or treat urinary incontinence and pelvic organ prolapse. These are: (1) women develop the ability to consciously contract the pelvic floor muscles before and during the increase of intraabdominal pressure; and 2) the strengthening of this muscle group can build support for the bladder and uretra ${ }^{6}$.

Due to the fact that most Pilates exercises are performed in conjunction with the recruitment of PF muscle fibers, many Pilates instructors believe that the method can produce a significant increase in the force or contractility of the muscles. Furthermore, if Pilates promotes an improvement in the functioning of the pelvic floor muscles (PFM), it may be an alternative for the treatment and prevention of pelvic floor dysfunction.

Urinary incontinence, pelvic organ prolapse and other manifestations of pelvic floor dysfunction are highly prevalent in women, especially in old age? These symptoms are associated with a number of factors that lead to damage to the integrity of the pudendal nerve or skeletal muscle fibers of the levator ani or coccygeus muscles ${ }^{8}$.

Due to the high prevalence of pelvic floor dysfunction among the elderly, the present study aimed to verify the influence of a protocol of Pilates exercises on the functionality and contractility of the PFM of elderly women.

\section{METHODS}

The study consisted of an initial sample of 30 volunteers who were enrolled at the Universidade Aberta para a Terceira Idade (the Open University for the Third Age) at the Federal University of Mato Grosso do Sul (UnAPI / UFMS), in the city of Campo Grande, Mato Grosso, Brazil. The subjects were invited to participate in the survey via telephone. However, eleven did not meet the inclusion criteria, six declined the invitation and three alleged personal reasons that prevented them from attending the exercise therapy sessions. The present study therefore adopted a longitudinal design, in which ten elderly women were accompanied during the execution of the procedure. The sample size calculation was based on the delimitation of type 1 error at $5 \%(\alpha=0.05)$, a power of $80 \%(1-\beta=0.20)$, under a bicaudal curve, and an effect size of 0.50 . Due to the lack of studies addressing the impact of Pilates on the PFM, it was not possible to use data published in literature to calculate the effect size. Thus, the delimitation of this topic was carried out using the hypothesis of improvement initially stipulated by the authors. From the calculations, the ideal sample size should be at least 28 subjects. This fact led us to an initial screening of 30 subjects, as shown in Figure 1.

The inclusion criteria involved healthy, sedentary women (normal weight, without psychiatric and/or neurological diseases), the Metabolic Equivalent of Task (MET) of whose self-reported daily activities did not exceed three times the baseline value. The women were aged over 60 years, sexually active, non-smokers, non-alcoholics, with no clinical diagnosis of pelvic floor dysfunction, no history 
of urinary tract infection, and no previous history of gynecological surgery (in previous five years) or neurological or endocrine-metabolic disorders. Women who were allergic to latex gloves or condoms or who did not complete three Pilates sessions were excluded from the study. Based on these criteria, the initial sample suffered considerable losses and included ten participants, as detailed in figure 1.

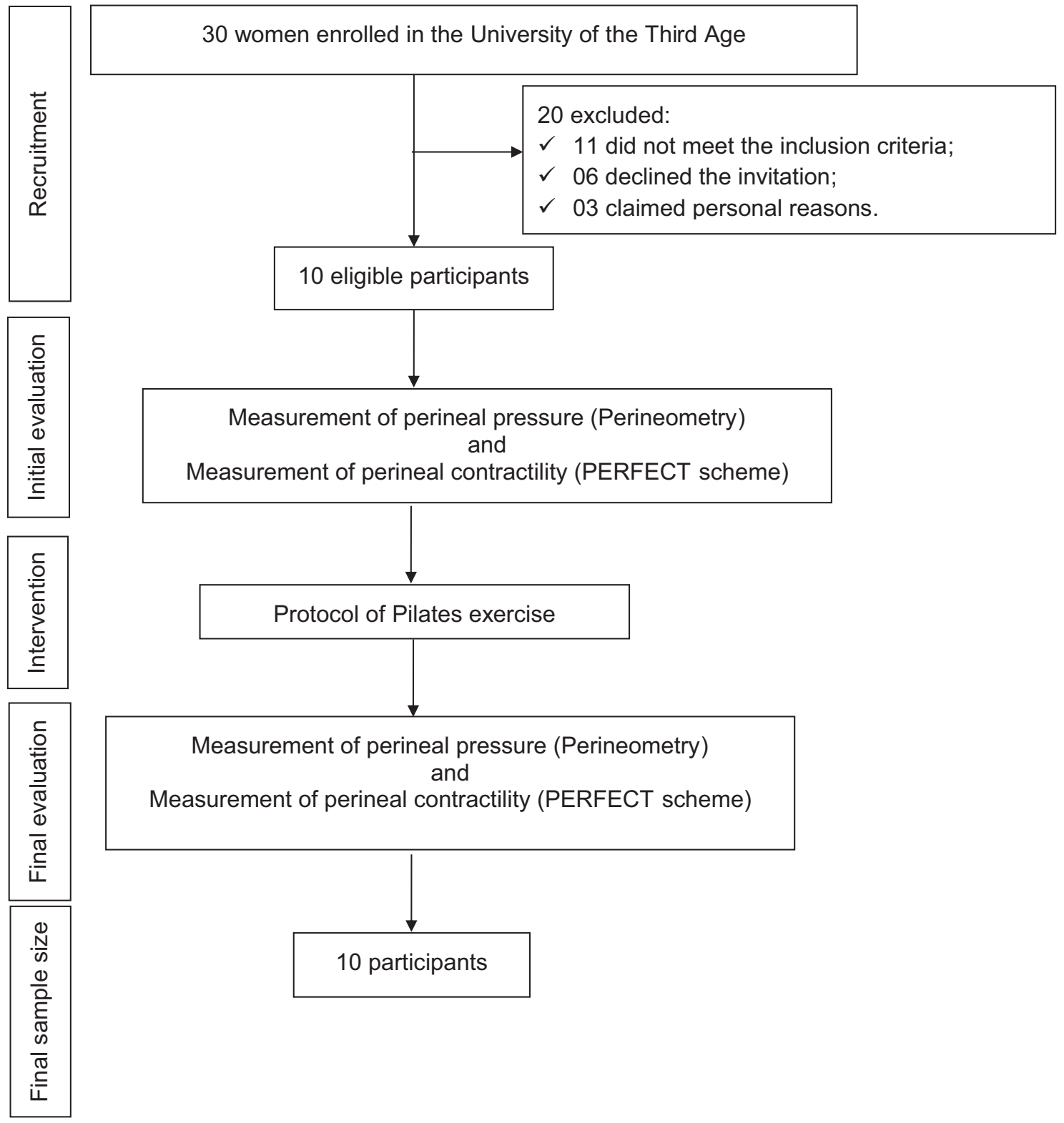

Figure 1. Study flow chart. Mato Grosso do Sul, 2015. 
The participants were previously informed about the data collection procedures, and all signed a free and informed consent form. Assessments and reassessments were performed by the same researcher. Anamnesis was performed initially, and soon after the pressure and contractility of the PFM were assessed, performed with the patient in the dorsal lithotomy position ${ }^{8}$.

The measurement of intracavitary perineal pressure was performed using a perineometer (Perina, QUARK, Piracicaba, São Paulo, Brazil), graduated from $0-48 \mathrm{~cm} \mathrm{H} 2 \mathrm{O}$. Perineometry is a pressure gauge which captures the pressure generated during the activation of the PFM, providing a reading on the display of the device on a scale in $\mathrm{cmH} 2 \mathrm{O}$. The equipment has an attached latex vaginal probe $(25 X 90 \mathrm{~cm})$ inflated by a pear type control ${ }^{9}$.

The PERFECT scheme consists of seven items and was developed and validated to assess the functionality of the $\mathrm{PFM}^{10}$. The "P" (power/force) indicator evaluates the intensity of the contraction of the PFM, graded using the modified Oxford scale. The "E" (endurance/resistance) result evaluates how long the contraction is maintained and sustained in seconds, until a loss of $35 \%$ or more. The "R" (repetitions) result assesses the number of repetitions of the sustained contraction (up to 10) that the participant is able to perform with satisfactory duration (5 seconds), with 4 seconds between each contraction. The "F" (fast/rapid contractions) result is evaluated after a short rest break ( 1 minute), and measures the number of contractions lasting one second each (up to ten contractions).

During the vaginal probe measurements the probe is coated a non-lubricated condom and inserted into the vaginal canal of the participant to capture the contractions. After the observing and recording of the intensity in $\mathrm{cmH} 2 \mathrm{O}$ of five maximal voluntary contractions, with a 5 second interval between each, the average was calculated. Perineal contractions were held during expiration, and the participants were instructed on the correct contractile movement of the PFM, avoiding the use of the accessory muscles.

There was a rest interval of two minutes between perineometry assessment and the evaluation of vaginal palpation. The participants were instructed to contract the PFM following the commands given by the examiner, which followed the steps determined by the PERFECT scheme and the intervals of rest ${ }^{10}$. All evaluations were performed before and after the 12-week follow-up period.

Chart 1 demonstrates the Pilates protocol realized in the present study. It consisted of 11 different Pilates postures: Pilates Breathing, Spine Stretch, Swan, Shoulder Bridge, Hundreds, Double Leg Stretch, Footwork, Roll up, Single Leg Stretch, Leg Pull Back, Kick front and back. It was performed twice a week, with each session lasting 60 minutes, for 12 weeks, totaling 24 sessions. The progression of the exercises was based on increasing the number of repetitions of the exercise, and variations in posture from beginner to intermediate and advanced, for each exercise. The movements were repeated six to eight times each.

Statistical analysis of the data was performed through descriptive and inferential analysis. Due to the non-parametric character of the data, the results were initially detailed as median \pm interquartile range. The Wilcoxon paired test was used for inferential analysis, while still respecting the nonparametric sum of the data. The delimitation of the null hypothesis (hypothesis of equality between pre- and post-intervention evaluations) in relation to the alternative hypothesis (hypothesis of difference between pre- and post-intervention evaluations) adopted a significance level of $5 \%(p<0.05)$.

Ethical approval was previously obtained from the institutional review board, and the research was registered under number 18879713.1.0000.0021. The study complied with Resolution 466/2012 of the Health Council of the Brazilian Ministry of Health. 
Chart 1. Exercise protocol applied to the subjects. Mato Grosso do Sul, 2015.

\begin{tabular}{|c|c|}
\hline Exercises (Week 1-12) & Description \\
\hline Pilates breathing & $\begin{array}{l}\text { Breathe slowly and concentrate on diaphragm movement. As air is exhaled, with pursed lips, } \\
\text { perform isometrics in the "power house". }\end{array}$ \\
\hline Spine Stretch & $\begin{array}{l}\text { Sitting to standing height, legs stretched out on the mat, knees slightly bent, chin to chest, roll, } \\
\text { flexing the trunk forward without losing the neutral position of the pelvis, forming the letter } \\
\text { "C" with the body, and return to starting position. }\end{array}$ \\
\hline Swan & $\begin{array}{l}\text { Prone, pelvis and spine neutral. Legs straight and lateral rotation. Bent arms next to the torso. } \\
\text { Inhale and stretch arms and raise the trunk. }\end{array}$ \\
\hline Shoulder Bridge & $\begin{array}{l}\text { Lying supine, legs bent and feet parallel. The movement is initiated by the activation of the } \\
\text { power house, followed by gluteal contraction, retroversion of the pelvis and lifting of the } \\
\text { pelvis. The return is targeted to the starting position. }\end{array}$ \\
\hline Hundred & $\begin{array}{l}\text { Lying supine, knees bent, feet flat on the floor, arms at sides. Inhale and raise the head and } \\
\text { trunk until reaching the base of the shoulder blades. Arms stretch forward as if reaching the } \\
\text { feet. Pump arms up and down with short, quick movements. Inhale for } 5 \text { pulses and perform } \\
\text { the cycle to complete } 100 \text { pulsations. }\end{array}$ \\
\hline Double leg stretch & $\begin{array}{l}\text { Lying supine, supported trunk, hips and knees bent, raise the cervical spine, hold the ankles } \\
\text { with the hands. Extend legs and arms simultaneously to } 45 \text { degrees and back to the starting } \\
\text { position, without letting the feet touch the ground. }\end{array}$ \\
\hline Footwork & $\begin{array}{l}\text { Standing, neutral pelvis, heels together, raise the body, resting the hands on a bar, bend the } \\
\text { knees and hips with hip external rotation, power house drive. Return to starting position. }\end{array}$ \\
\hline Roll up & $\begin{array}{l}\text { Supine, both arms extended above the head and with the knees bent. Participants must move } \\
\text { from lying to sitting, arms extending toward the feet, flexing the trunk and holding the arms } \\
\text { outstretched. A deep breath should be taken at the beginning of preparation and exhaled when } \\
\text { starting the roll-up. Return to the original position. }\end{array}$ \\
\hline Single leg stretch & $\begin{array}{l}\text { Lying supine raise the shoulders and keep the chin toward the chest, raise one leg and hold the } \\
\text { ankle, return to the starting position and switch legs. }\end{array}$ \\
\hline Leg pull back & $\begin{array}{l}\text { Fours on the ground in the prone position. Align hands/elbows to hips. Legs at the width of } \\
\text { the hips. Extend one leg at a time. Inhale as lift leg and exhale while returning to the ground. }\end{array}$ \\
\hline Kick front and back & $\begin{array}{l}\text { In the lateral position with the lower arm extended above the head. Position the other arm to } \\
\text { the front of the torso, resting the palm on the mat. Lift the leg from the mat and flex the hip, } \\
\text { placing the hallux on the mat twice. Extend the leg and repeat. }\end{array}$ \\
\hline
\end{tabular}

\section{RESULTS}

The present study included ten women duly enrolled in UnAPI/UFMS, who were aged $63.4 \pm 4.5$ years at entry. Table 1 details the general characteristics of the participants.

Table 2 shows the results of the comparative analysis of the intensity of PFM pressure and contractility before and after 12-weeks of follow-up. The results indicate an increase in PFM pressure and contractility ( $p=0.012)$. Regarding the PERFECT scheme, the benefits of the intervention was observed in the endurance $(p=0.017)$ and number of fast repetitions $(p=0.008)$ variables. No apparent benefit of the Pilates method was observed in the power $(p=0.157)$ and number of slow repetitions variables $(p=0.156)$. 
Table 1. General characteristics of the participants. Mato Grosso do Sul, 2015.

\begin{tabular}{ll}
\hline Variables & Prevalence $(\%)$ \\
\hline Final sample size & $10(100.0)$ \\
\hline Marital status & $0(0.0)$ \\
Single / divorced & $7(70.0)$ \\
Married & $3(30.0)$ \\
Widowed & \\
\hline Children & $8(80.0)$ \\
0 & $0(0.0)$ \\
1 to 3 & $2(20.0)$ \\
More then 3 & \\
Hormone replacement therapy & $1(10.0)$ \\
Yes & $9(90.0)$ \\
No & \\
\hline Loss of urine when coughing & $2(20.0)$ \\
Yes & $8(80.0)$ \\
No & \\
Daytime urinary urgency & $1(10.0)$ \\
Yes & $9(90.0)$ \\
No & \\
\hline
\end{tabular}

Table 2. Initial and final values of the participants regarding maximal voluntary contraction and the PERFECT scheme. Mato Grosso do Sul, 2015.

\begin{tabular}{llll}
\hline Variables & $\begin{array}{l}\text { Initial values } \\
\text { (median } \pm \text { interquartile range) }\end{array}$ & $\begin{array}{l}\text { Final values } \\
\text { (median } \pm \text { interquartile range) }\end{array}$ & $P$ \\
\hline Perineometry $\left(\mathrm{cm} \mathrm{H}_{2} \mathrm{O}\right)$ & $79.0 \pm 83.5$ & $90.0 \pm 82.0$ & .012 \\
P - Power & $3.0 \pm 1.0$ & $3.0 \pm 1.0$ & .157 \\
E - Endurance & $5.0 \pm 0.1$ & $7.0 \pm 4.7$ & .017 \\
R - Repetitions & $3.0 \pm 2.5$ & $5.5 \pm 3.2$ & .156 \\
F - Fast contractions & $7.0 \pm 4.5$ & $8.0 \pm 4.7$ & .008 \\
\hline
\end{tabular}

Inferential analysis was performed using the Wilcoxon test.

\section{DISCUSSION}

The subjects of the present study were elderly women (median age of $63.4 \pm 4.5$ years) with little or no pelvic floor dysfunction, who were all nonsmokers and had few children (average number of children of two). These inclusion criteria were proposed to volunteers who had one muscle in the entire PF which could respond to training ${ }^{11}$.
Two recent Cochrane systematic reviews have recommended the training of the PFM through Kegel exercises as the first line of conservative treatment for urinary incontinence, urgency and mixed as well as fecal incontinence ${ }^{12,13}$. However, PF training with Kegel exercises requires proper instruction to be effective and a long follow-up period for the results to be maintained. Glazener et al. ${ }^{14}$ described improvement of the symptoms of pelvic floor dysfunction after 
childbirth by training with Kegel exercises among a group of highly motivated women. However, the improvement did not persist due in part to the loss of adherence to the exercise program over time. Other authors likewise report major short-term benefits provided by Kegel exercises, but also describe a low compliance rate in the long-term ${ }^{15,16}$.

The study by Sluijs et al. ${ }^{17}$ described greater adherence among patients to training programs which featured positive feedback and a shorter interruption in activities of their daily routine. Thus, it can perhaps be inferred that Pilates training that provides positive feedback and is easily adapted to the individual's routine is necessary to keep patients motivated. These exercises promote flexibility, the strengthening of the entire body and produce greater physical fitness and body and mind integration ${ }^{18,19}$.

Due to the fact that the activation of the "core" recruits the deep muscles of the pelvis, the present study identified improved functionality and contractility of the PFM after training with the proposed protocol. Thus, older women can benefit from the comprehensive training that this technique provides to improve PF functionality, and thus prevent the PF distortion that is so common in older women.

Cullingan et al. ${ }^{20}$ compared changes in the strength of the pelvic floor muscles achieved by young women after a 12 -week Pilates program or a 12 -week program of Kegel exercises. Both groups achieved significant increases in the intensity of PFM pressure in $\mathrm{cmH} 2 \mathrm{O}$, with no difference between the groups. It was found that Pilates was as effective at promoting the strengthening of the PFM as the traditional exercises developed by Kegel ${ }^{21}$.

However, a study that evaluated young women who performed Pilates and non-Pilates practitioners found no difference between the two groups regarding maximum voluntary contraction $(\mathrm{cmH} 20)$ and standardized vaginal palpation through the PERFECT scheme ${ }^{21}$. It is believed that the divergence of results found can be justified by the findings of a FERLA study that assessed contractility and PFM functionality among women who practiced Pilates. In this study, PFM assessments were carried out before the initial voluntary training with the proposed protocol. During the functional evaluation of the pelvic floor, the volunteer was instructed how to correct the PFM without the interference of the accessory muscles, so promoting information and body awareness. The volunteer therefore uses this knowledge during the exercises, especially in the recruitment of PFM which occurs during the activation of the "powerhouse" for the effectiveness of the technique.

A randomized controlled trial ${ }^{22}$, with the evaluation of PF prior to treatment, compared the Pilates method with a Kegel exercise program, and demonstrated the effectiveness of the Pilates method to treat male urinary incontinence.

Evaluation of the PF is essential for the development of appropriate treatment and the monitoring of results. There is currently no assessment tool that is considered gold standard, though the ICS recommends that the evaluation of PFM is carried out through vaginal palpation and perineometry, among others ${ }^{23}$, as was performed in this study.

Functional assessment of prior pelvic floor training is generally not performed in Pilates studios or clinics that use this method in physical therapy. It would be extremely useful if the objectives of such training included the strengthening of the deep pelvic muscles. It is common for healthy women to fail to properly contract the PFM, as they are internal, deep location, muscles, and are not always recruited during physical activities ${ }^{24}$.

It is important to consider some limitations of this study. The small sample size is due to the fact that some of the women did not meet the "being sexually active" inclusion criteria of the study while another individual refused to participate in the evaluation because of shame or embarrassment. Another possible criticism of this study could be the absence of a control group, but it is believed that it would be impossible to provide a credible Pilates placebo treatment protocol.

Finally, the present study indicates the need for further studies with symptomatic patients, adapting the Pilates protocol for women with urinary incontinence and other PFM disorders. 


\section{CONCLUSION}

Training with the exercises devised by Joseph Pilates increased the pressure resistance and the number of rapid contractions of the pelvic floor muscles. The results of the present study are encouraging and may eventually lead to enhanced therapeutic Pilates use to treat or prevent disorders of the pelvic floor, especially in periods in which these muscles are most required, such as pregnancy, vaginal postpartum or in old age.

\section{REFERENCES}

1. Rahimimoghadam Z, Rahemi Z, Mirbagher Ajorpaz N, Sadat Z. Effects of Pilates exercise on general health of hemodialysis patients. J Bodyw Mov Ther. 2017;21(1):86-92.

2. Dos Santos NT, Raimundo KC, da Silva SA, Souza LA, Ferreira KC, Borges Santo Urbano ZF, Gasparini AL, Bertoncello D. Increased strength of the scapular stabilizer and lumbar muscles after twelve weeks of Pilates training using the Reformer machine: A pilot study. J Bodyw Mov Ther. 2017 Jan;21(1):74-80.

3. Oliveira LC, Pires-Oliveira DA, Abucarub AC, Oliveira LS, Oliveira RG.Pilates increases isokinetic muscular strength of the elbow flexor and extensor muscles of older women: A randomized controlled clinical trial.J Bodyw Mov Ther. 2017;21(1):2-10.

4. Vasconcelos AP, Cardozo DC, Lucchetti AL, Lucchetti G.Comparison of the effect of different modalities of physical exercise on functionality and anthropometric measurements in community-dwelling older women. J Bodyw Mov Ther. 2016;20(4):851-6.

5. Josephs S, Pratt ML, Calk Meadows E, Thurmond S, Wagner A. The effectiveness of Pilates on balance and falls in community dwelling older adults. J Bodyw Mov Ther. 2016;20(4):815-23.

6. Bo K Pelvic floor muscle training in treatment of female stress urinary incontinence, pelvic organ prolapse and sexual dysfunction.World J Urol. 2012;30(4):437-43.

7. Bazi T, Takahashi S, Ismail S, Bø K, Ruiz-Zapata AM, Duckett J, Kammerer-Doak D. Prevention of pelvic floor disorders: international urogynecological association research and development committee opinion. Int Urogynecol J. 2016;27(12):1785-95.

8. Souza CEC et al. Estudo comparativo da função do assoalho pélvico em mulheres continentes e incontinentes na pós menopausa. Rev Bras Fisioter. 2009; 13(6):535-41.
9. Hundley AF, Wu JM, Visco AG. A comparison of perineometer to brink score for assessment of pelvic floor muscle strength. Am J Obstet Gynecol. 2005; 192:1583-91.

10. Laycock J, Jerwood D. Pelvic floor assessment; the PERFECT scheme. Physiotherapy. 2001; 87(12):631-42.

11. Alperin M, Cook M, Tuttle LJ, Esparza MC, Lieber RL. Impact of vaginal parity and aging on the architectural design of pelvic floor muscles. Am J Obstet Gynecol. 2016;215(3):312.e1-9.

12. Dumoulin C, Hay-Smith EJC, Habée-Séguin GM. Pelvic floor muscle training versus no treatment, or inactive control treatments, for urinary incontinence in women. Cochrane library. 2014. doi:10.1002/14651858.CD005654

13. Hay-Smith J, Morked S, Fairbrother KA, Herbison GP. Pelvic floor muscle training for prevention of urinary and faecal incontinence in antenatal and postnatal women. Cochrane Database Syst Rev 8(4):CD007471, 2008.

14. Glazner LM, Herbison GP, MacArthur C, Grant A, Wilson PD. Randomised controlled trial of conservative management of potential urinary and fecal incontinence: six year follow up. BMJ. 2005 Feb $12 ; 330(7487): 337 .$.

15. Torella M, Schettino MT, Tammaro C, Grimaldi A, Del Deo F, Gimigliano F, Colacurci N Longterm outcomes of perineal rehabilitation. Minerva Ginecol. 2014;66(2):219-27.

16. Pereira VS, de Melo MV, Correia GN, Driusso P. Long-term effects of pelvic floor muscle training with vaginal cone in post-menopausal women with urinary incontinence: a randomized controlled trial. Neurourol Urodyn. 2013;32(1):48-52.

17. Sluijs E, Kok G, Zoe J. Correlates of exercise compliance in physical therapy. Phys Ther. 1993;73(11):771-82. 
18. Lately P. Updating the principles of the Pilates method-Part 2. J Bodyw Mov Ther. 2002; 6(2):94-101.

19. Granacher U, Gollhofer A, Hortobágyi T, Kressig RW, Muehlbauer T. The importance of trunk muscle strength for balance, functional performance, and fall prevention in seniors: a systematic review. Sports Med. 2013;43(7):627-41.

20. Culligan PJ, Scherer J, Dyer K, Priestley JL, GuingonWhite G, Delvecchio D, Vangeli M. A randomized clinical trial comparing pelvic floor muscle training to a Pilates exercise program for improving pelvic muscle strength. Int Urogynecol J. 2010;21(4):401-8

21. Ferla L, Paiva LL, Darki C, Vieira A. Comparison of the functionality of pelvic floor muscles in women who practice the Pilates method and sedentary women: a pilot study. Int Urogynecol J. 2016 Jan;27(1):123-8.

Received: October 20, 2016

Reviewed: April 21, 2017

Accepted: June 01, 2017
22. Pedriali FR, Gomes CS, Soares L, Urbano MR, Moreira EC, Averbeck MA, de Almeida SH. Is pilates as effective as conventional pelvic floor muscle exercises in the conservative treatment of postprostatectomy urinary incontinence? A randomised controlled trial. Neurourol Urodyn. 2016;35(5):615-21.

23. Staskis D, Kelleher C, Avery K. Initial assessment of urinary and faecal incontinence in adult male and female patients. In: Abrams P, Cardozo L, Wein A, Khoury S, editors. Incontinence: 4th International Consultation on Incontinence. Paris, France: Health Publications; 2009. p. 311-412.

24. Hilde G, Stær-Jensen J, Ellström Engh M, Brækken $\mathrm{IH}, \mathrm{B} ø \mathrm{~K}$. Continence and pelvic floor status in nulliparous women at midterm pregnancy. Int Urogynecol J. 2012;23(9):1257-63. 